\title{
Synthesis, NMR, X-ray crystallography and bioactivity of some $\alpha$-aminophosphonates
}

\author{
Gandavaram Syam Prasad, ${ }^{a}$ Jadaprolu Radha Krishna, ${ }^{b}$ Manubolu Manjunath, \\ Obulam Vijaya Sarathi Reddy, ${ }^{\mathrm{c}}$ Musali Krishnaiah, ${ }^{\mathrm{b}}$ Cirandur Suresh Reddy, ${ }^{*}, \mathrm{a}$ and \\ Vedavati G. Puranik ${ }^{d}$ \\ ${ }^{a}$ Department of Chemistry, ${ }^{b}$ Physics, ${ }^{c}$ Biochemistry, Sri Venkateswara University, \\ Tirupati-517 502, India \\ ${ }^{d}$ Centre of Material Characterization, National Chemical Laboratory, Pune-411 008, India \\ E-mail:csureshsvu@yahoo.com
}

\begin{abstract}
The synthesis of some new $\alpha$-aminophosphonates (4a-l) was accomplished by one pot reaction of equimolar quantities of 2-amino-4-chlorophenol (1), various aromatic aldehydes (2a-l) and diethylphosphite (3) in dry toluene at reflux temperature. Products $\mathbf{4 a - l}$ were characterized by IR, ${ }^{1} \mathrm{H}$, ${ }^{13} \mathrm{C},{ }^{31} \mathrm{P} \mathrm{NMR}$ and in the case of $\mathbf{4 g}$ by X-ray crystal diffraction data.
\end{abstract}

Keywords: $\alpha$-Aminophosphonates, antibacterial

\section{Introduction}

Organophosphorus compounds have found a wide range of application in the areas of industry, agriculture and medicinal chemistry owing to their unique physical and biological properties. Their utility in organic synthesis as reagents and potential synthons is gaining increased attention. ${ }^{1}$

The synthesis of $\alpha$-aminophosphonates has attracted much interest because of their biological activity and structural analogy to $\alpha$-amino acids. ${ }^{2}$ They also act as peptide mimics, ${ }^{3}$ enzyme inhibitors, ${ }^{4}$ haptens of catalytic antibodies, ${ }^{5}$ antibiotics and pharmacological agents ${ }^{6}$ and the analogues of amino acids, aminophosphonic, and aminophosphinic acids are of considerable interest due to their properties in agrochemistry as plant growth regulators and herbicides. ${ }^{7}$ As a result, a variety of synthetic approaches ${ }^{8}$ have been developed for the synthesis of $\alpha$ aminophosphonates. Of these methods, the nucleophilic addition of phosphates with imines, catalyzed by an acid or a base is one of the most convenient methods. In our work we synthesized $\alpha$-aminophosphonates under mild conditions without catalyst. 


\section{Results and Discussion}

The synthetic route for the title compounds (4a-l) involves reaction of equimolar quantities of 2 amino-4-chlorophenol (1), various aromatic aldehydes (2a-1) and diethylphosphite (3) in toluene at reflux temperature (Scheme-1). The chemical structures of all the new compounds are confirmed by elemental analysis, IR, ${ }^{1} \mathrm{H}$ NMR, ${ }^{13} \mathrm{C}$ NMR and ${ }^{31} \mathrm{P}$ NMR spectral data. Compounds 4a-I exhibited characteristic IR stretching frequencies in the region ${ }^{9} 1191-1264,742-758$ and 3274$3404 \mathrm{~cm}^{-1}$ for $\mathrm{P}=\mathrm{O}, \mathrm{P}-\mathrm{C}_{(\text {aliphatic) }}$ and $\mathrm{N}-\mathrm{H}$ respectively.

The aromatic protons of the two benzene rings of $\alpha$-aminophosphonates $(\mathbf{4 a - 1})$ showed a complex multiplet at $\delta 6.41-7.51$. The P-C-H proton signal appeared as multiplet ${ }^{10 a}$ at $\delta 4.09$ 4.19 due to its coupling with phosphorus and proton of N-H. The N-H proton signal appeared at $\delta$ 4.83-4.99 as doublet $(J=23.6-24.4 \mathrm{~Hz})$ for the compounds $\mathbf{4 a - c}, \mathbf{4 h -} \mathbf{i}$ and $\mathbf{4} \mathbf{l}$ and as a singlet in compounds $\mathbf{4 d - g}$ and $\mathbf{4 j - k}$. The methylene protons of $\mathrm{POC}_{2} \mathrm{CH}_{3}$ showed a multiplet and methyl protons of $\mathrm{POCH}_{2} \mathrm{CH}_{3}$ showed a triplet in the region 3.79-4.09 and 1.11-1.66 ppm respectively. ${ }^{10}$ The carbon chemical shifts for P-C-H, P-O- $\mathrm{CH}_{2}-\mathrm{CH}_{3}$ and $\mathrm{P}-\mathrm{OCH}_{2}-\mathrm{CH}_{3}$ in the title compounds observed in the expected region. ${ }^{10 \mathrm{~b}}{ }^{31} \mathrm{P}$ NMR signals ${ }^{11}$ appeared in the region $22.30-27.44 \mathrm{ppm}$ for all the compounds $\mathbf{4 a - 1 .}$

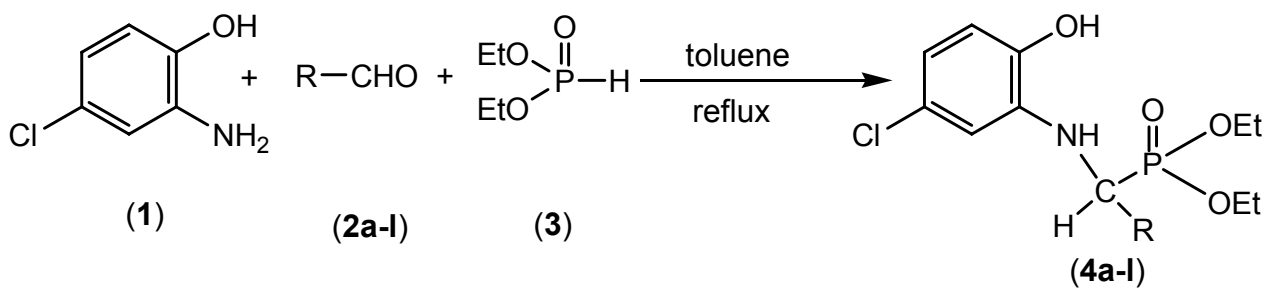

\begin{tabular}{llll} 
Compd. & \multicolumn{1}{c}{$\mathbf{R}$} & Compd. & \multicolumn{1}{c}{$\mathbf{R}$} \\
2a \& 4a & $4-\mathrm{FC}_{6} \mathrm{H}_{4}$ & 2g \& 4g & $3,4-(\mathrm{OMe})_{2} \mathrm{C}_{6} \mathrm{H}_{3}$ \\
2b \& 4b & $4-\mathrm{ClC}_{6} \mathrm{H}_{4}$ & $\mathbf{2 h ~ \& ~ 4 h}$ & $\mathrm{C}_{6} \mathrm{H}_{5}$ \\
2c \& 4c & $4-\mathrm{BrC}_{6} \mathrm{H}_{4}$ & $\mathbf{2 i ~ \& ~ 4 i}$ & $\mathrm{C}_{4} \mathrm{H}_{3} \mathrm{~S}$ \\
2d \& 4d & $4-\mathrm{MeC}_{6} \mathrm{H}_{4}$ & $\mathbf{2 j}$ \& 4j & $3-\mathrm{OH}-4-\mathrm{OMeC}_{6} \mathrm{H}_{3}$ \\
2e \& 4e & $4-\mathrm{AllyloxyC}_{6} \mathrm{H}_{4}$ & $\mathbf{2 k}$ \& 4k & $3-\mathrm{OHC}_{6} \mathrm{H}_{4}$ \\
2f \& 4f & $4-\mathrm{OMeC}_{6} \mathrm{H}_{4}$ & $\mathbf{2 l ~ \& ~ 4 l}$ & $2-\mathrm{OHC}_{6} \mathrm{H}_{4}$
\end{tabular}

\section{Scheme 1}

\section{X-ray crystal structure determination of $\mathbf{4 g}$}

Intensity data were collected on a Siemens SMART CCD diffractometer with monochromated Mo-K $\alpha$ radiation $(\lambda 0.7107 \AA)$. Structures were solved by direct methods and routinely developed and refined (on $F^{2}$ ) using the SHELX-97 programs. ${ }^{12}$ Transparent colourless crystals were obtained from methanol. Crystal data: $\mathrm{C}_{19} \mathrm{H}_{25} \mathrm{ClNO}_{6} \mathrm{P}, \mathrm{M}_{\mathrm{r}} 429.82 \mathrm{gmol}^{-1}$, monoclinic, space group $\mathrm{P} 2(1) / \mathrm{n}, \mathrm{a}=13.8079(19), \mathrm{b}=9.7872(14), \mathrm{c}=16.907(2) \AA, \beta=103.832(2)^{\circ}$, 
$\mathrm{V}=2218.6(5) \AA^{3}, D_{\text {calc }}=1.287 \mathrm{~g} \mathrm{~cm}^{-3}, \mathrm{Z}=4, \mu(\mathrm{Mo} \mathrm{K} \alpha)=0.277 \mathrm{~mm}^{-1}$, Size $0.16 \times 0.19 \times 0.53$ $\mathrm{mm}, F(000)=904, T=293 \mathrm{~K}$. Total data 20878 , unique data $3912\left(R_{\text {int }}=0.0208\right)$ in the range $2.18^{\circ} \leq \theta \leq 25^{\circ}, 3912$ with $I \geq 2 \sigma(I)$, empirically corrected for absorption and other effects. All non-H atoms anisotropic: $\mathrm{H}$ atoms in calculated positions. $R_{\mathrm{I}}(I>2 \sigma(I))=0.0705, w R 2$ (all data) $0.2224, \mathrm{GoF}=1.071$, largest residual feature $|1.154| \mathrm{e} \AA^{-3}$. The structure is illustrated in Figure 1. Full details of the structure determinations have been deposited with the Cambridge Crystallographic Data Centre as CCDC- 639459 (4g). Copies of this information may be obtained free of charge from The Director, CCDC, 12 Union Road, Cambridge CB2 1EZ, UK (fax: +44 1223336 033; e-mail: deposit@ccdc.cam.ac.uk or www: http//www.ccdc.cam.ac.uk).

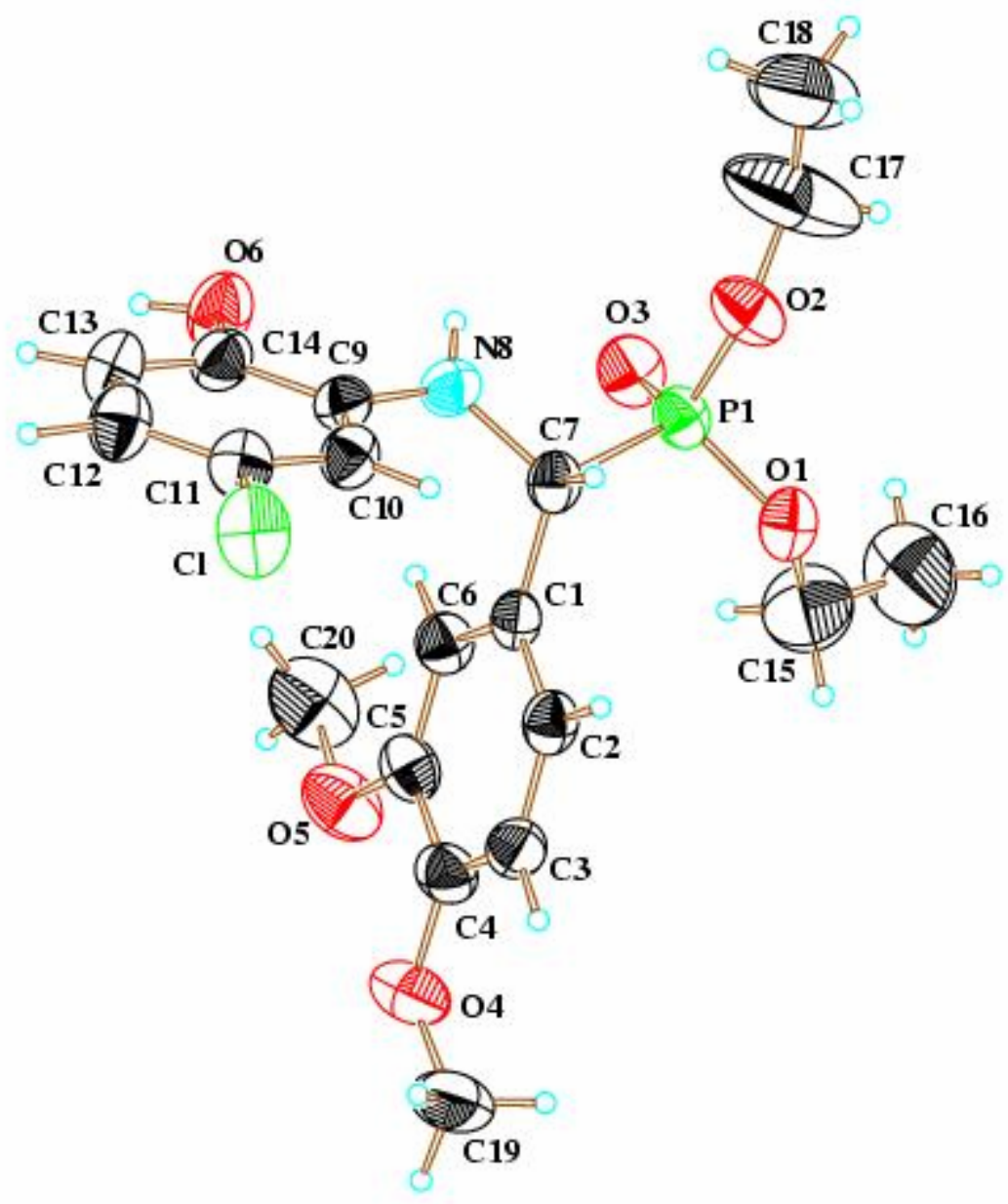

Figure 1. Molecular structures of $\mathbf{4 g}$ showing the atom-labelling scheme

The O3-P1-O1 $\left[115.4(2)^{\circ}\right]$, O3-P1-O2 [115.1(2) $\left.{ }^{\circ}\right]$ and O3-P1-C7 $\left[112.5(1)^{\circ}\right]$ bond angles are larger than $\mathrm{O} 1-\mathrm{P} 1-\mathrm{O} 2\left[102.6(2)^{\circ}\right], \mathrm{O} 1-\mathrm{P} 1-\mathrm{C} 7\left[106.4(1)^{\circ}\right]$ and $\mathrm{O} 2-\mathrm{P} 1-\mathrm{C} 7\left[103.6(4)^{\circ}\right]$ bond angles indicating a distorted tetrahedral configuration for the phosphorus atom. The deformation of the $\mathrm{PO}_{3} \mathrm{C}$ tetrahedron in this molecule may be due to the steric effects of ethoxy groups in which their atoms exist in distorted conformation. The $\mathrm{C}\left(\mathrm{sp}^{3}\right)-\mathrm{N}$ bond length $1.445(4) \AA$ is 
intermediate between the normal C-N $\operatorname{single}^{13}[1.47$ (1) $\AA]$ and double bonds ${ }^{14}$ [1.28 (1) $\left.\AA\right]$ which is indicative of its partial double bond character. The interplanar angle between the aniline moiety and the phenyl ring bonded to the stereogenic center $\mathrm{C} 7$ is $83.7(1)^{\circ}$. The methoxy groups are almost coplanar with the benzene ring to which they are substituted, with a $\mathrm{C} 6-\mathrm{C} 5-\mathrm{O} 5-\mathrm{C} 20$ \& $\mathrm{C} 3-\mathrm{C} 4-\mathrm{O} 4-\mathrm{C} 19$ torsion angles of $4.8(6)^{\circ} \&-1.6(5)^{\circ}$. Chloro and hydroxy groups are also coplanar with aniline moiety. In the crystal structure, molecules are linked by intramolecular $\mathrm{N}-$ $\mathrm{H} \cdots \mathrm{O}$ and intermolecular $\mathrm{C}-\mathrm{H} \cdots \mathrm{O}$ and $\mathrm{O}-\mathrm{H} \cdots \mathrm{O}$ hydrogen bonds.

\section{Antibacterial activity}

The compounds were diluted in DMSO for bioassay. Solvent control was included although no antibacterial activity has been noted for the solvent employed. Ciprofloxacin (Hi-media) controls were included to compare its activity with that of 4a-l. All samples were tested in triplicate and average results were recorded.

The compounds were assayed for antibacterial activity against six registered bacterial isolates which were obtained from the NCIM (National Collection of Industrial Microorganisms, National Chemical Laboratories, Pune-411 003, India). The bacteria included Gram positive bacterial isolates-Staphylococcus aureus (NCIM No: 5021, ATCC No. 25923), Bacillus subtilis (NCIM No: 2063, ATCC No. 6633) and four Gram negative bacteria-Escherichia coli (NCIM No: 2931, ATCC No. 25922), Pseudomonas aeruginosa (NCIM No: 5029, ATCC No: 27853), Salmonella typhimurium (NCIM No: 2501, ATCC No: 23564) and Klebsiella pneumoniae (NCIM No: 2957). The bacteria were grown on Hi-media nutrient agar and sub cultured as needed.

\section{Experimental Section}

General Procedures. Chemicals procured were of commercial quality and were distilled / recrystallised before use. Elemental analyses were performed by the Central Drug Research Institute, Lucknow, India. IR spectra were recorded as $\mathrm{KBr}$ pellets or Nujol mulls on a Perkin Elmer 283 unit. The ${ }^{1} \mathrm{H},{ }^{13} \mathrm{C}$ and ${ }^{31} \mathrm{P}$ NMR spectra were taken on AMX $400 \mathrm{MHz}$ spectrometer operating at 400 $\mathrm{MHz}$ for ${ }^{1} \mathrm{H}, 100 \mathrm{MHz}$ for ${ }^{13} \mathrm{C}$ and $161.9 \mathrm{MHz}$ for ${ }^{31} \mathrm{P}$. All these compounds were dissolved in methanol- $d_{4}$. The chemical shifts in $\delta$ were referenced to TMS $\left({ }^{1} \mathrm{H}\right.$ and $\left.{ }^{13} \mathrm{C}\right)$ and $85 \% \mathrm{H}_{3} \mathrm{PO}_{4}\left({ }^{31} \mathrm{P}\right)$.

\section{General experimental procedure for products $4 a-1$}

To a stirred solution of 2-amino-4-chlorophenol (1) (0.72 g, $0.005 \mathrm{~mol})$ various aromatic aldehydes (2a-l) $(0.005 \mathrm{~mol})$ in anhydrous toluene $(15 \mathrm{~mL})$ was added dropwise. Stirring was continued at room temperature for $1 \mathrm{~h}$. Then diethylphosphite (3) $(0.7 \mathrm{~g}, 0.005 \mathrm{~mol})$ in anhydrous toluene $(15 \mathrm{~mL})$ was added dropwise. Stirring was continued at room temperature for another $0.5 \mathrm{~h}$, later the mixture was heated under reflux for 4-6 h. After completion of reaction (monitored by TLC) and the solvent was removed under reduced pressure. The resulting residue 
was purified by column chromatography on silica gel using petroleum ether-ethylacetate (8:2) as eluent. The spectral and analytical data for these compounds are presented below.

[(5-Chloro-2-hydroxyphenylamino)-(4-fluorophenyl)methyl]diethylphosphonate (4a). Yield (\%) : 65. mp : $145-147^{\circ} \mathrm{C}$. IR : $743\left(\mathrm{P}-\mathrm{C}_{\text {aliphatic }}\right), 1217(\mathrm{P}=\mathrm{O}), 3379(\mathrm{~N}-\mathrm{H}) \mathrm{cm}^{-1} .{ }^{1} \mathrm{H}$ NMR : $\delta 1.28$ (t, $\left.J=7.0 \mathrm{~Hz}, 3 \mathrm{H}, \mathrm{P}-\mathrm{OCH}_{2} \underline{\mathrm{C}}_{3}\right), 1.18$ (t, $J=7.0 \mathrm{~Hz}, 3 \mathrm{H}, \mathrm{P}-\mathrm{OCH}_{2} \mathrm{C}_{3}$ ), 3.86-4.04 (m, 4H, P$\left.\mathrm{OC}_{2} \mathrm{CH}_{3}\right), 4.13-4.17(\mathrm{~m}, 1 \mathrm{H}, \mathrm{P}-\mathrm{CH}), 4.98(\mathrm{~d}, J=24.1 \mathrm{~Hz}, 1 \mathrm{H}, \mathrm{NH}), 6.47(\mathrm{~d}, J=2.1 \mathrm{~Hz}, 2 \mathrm{H}$, Ar-H), 6.60 (d, $J=8.9 \mathrm{~Hz}, 1 \mathrm{H}, \mathrm{Ar}-\mathrm{H}), 7.08$ (t, $J=8.6 \mathrm{~Hz}, 2 \mathrm{H}, \mathrm{Ar}-\mathrm{H}), 7.47$ (t, $J=2.8 \mathrm{~Hz}, 2 \mathrm{H}, \mathrm{Ar}-$ H). ${ }^{31} \mathrm{P}$ NMR: $\delta$ 22.88. Anal. Calcd for $\mathrm{C}_{17} \mathrm{H}_{20} \mathrm{ClFNO}_{4} \mathrm{P}: \mathrm{C}, 52.34 ; \mathrm{H}, 5.20 ; \mathrm{N}, 3.61$. Found : $\mathrm{C}$, $52.16 ; \mathrm{H}, 5.15 ; \mathrm{N}, 3.54$.

[(5-Chloro-2-hydroxyphenylamino)-(4-chlorophenyl)methyl]diethylphosphonate (4b). Yield (\%) : 71. mp : $161-163^{\circ} \mathrm{C}$. IR : $744\left(\mathrm{P}-\mathrm{C}_{\text {aliphatic }}\right), 1199(\mathrm{P}=\mathrm{O}), 3399(\mathrm{~N}-\mathrm{H}) \mathrm{cm}^{-1} .{ }^{1} \mathrm{H}$ NMR : $\delta 1.28$ $\left(\mathrm{t}, J=7.0 \mathrm{~Hz}, 3 \mathrm{H}, \mathrm{P}-\mathrm{OCH}_{2} \underline{\mathrm{C}}_{3}\right), 1.18\left(\mathrm{t}, J=7.0 \mathrm{~Hz}, 3 \mathrm{H}, \mathrm{P}-\mathrm{OCH}_{2} \underline{\mathrm{C}}_{3}\right), 3.88-4.05(\mathrm{~m}, 4 \mathrm{H}$, P-OCH $\left.\underline{H}_{2} \mathrm{CH}_{3}\right), 4.12-4.17(\mathrm{~m}, 1 \mathrm{H}, \mathrm{P}-\mathrm{CH}), 4.99$ (d, $\left.J=24.4 \mathrm{~Hz}, 1 \mathrm{H}, \mathrm{NH}\right), 6.45$ (t, $J=2.0 \mathrm{~Hz}, 1 \mathrm{H}$, Ar-H), 6.47 (d, $J=2.1 \mathrm{~Hz}, 1 \mathrm{H}, \mathrm{Ar}-\mathrm{H}), 6.61$ (d, $J=8.1 \mathrm{~Hz}, 1 \mathrm{H}, \mathrm{Ar}-\mathrm{H}), 7.34$ (d, $J=8.3 \mathrm{~Hz}, 2 \mathrm{H}$, Ar-H), 7.43 (dd, $J=6.5,2.0 \mathrm{~Hz}, 2 \mathrm{H}, \mathrm{Ar}-\mathrm{H}) .{ }^{31} \mathrm{P} \mathrm{NMR}: \delta 22.59$. Anal. Calcd for $\mathrm{C}_{17} \mathrm{H}_{20} \mathrm{Cl}_{2} \mathrm{NO}_{4} \mathrm{P}$ : C, 50.51; H, 4.99; N, 3.47. Found : C, 50.32; H, 4.93; N, 3.40.

[(5-Chloro-2-hydroxyphenylamino)-(4-bromophenyl)methyl]diethylphosphonate (4c). Yield (\%) : 78. mp : $188-190^{\circ} \mathrm{C}$. IR : $743\left(\mathrm{P}-\mathrm{C}_{\text {aliphatic }}\right), 1213(\mathrm{P}=\mathrm{O}), 3399(\mathrm{~N}-\mathrm{H}) \mathrm{cm}^{-1} .{ }^{1} \mathrm{H}$ NMR : $\delta 1.28$ $\left(\mathrm{t}, J=7.0 \mathrm{~Hz}, 3 \mathrm{H}, \mathrm{P}-\mathrm{OCH}_{2} \underline{\mathrm{C}}_{3}\right), 1.19\left(\mathrm{t}, J=7.0 \mathrm{~Hz}, 3 \mathrm{H}, \mathrm{P}-\mathrm{OCH}_{2} \underline{\mathrm{C}}_{3}\right), 3.88-4.05(\mathrm{~m}, 4 \mathrm{H}$, P-OC $\underline{H}_{2} \mathrm{CH}_{3}$ ), 4.11-4.17 (m, 1H, P-CH), 4.98 (d, $\left.J=24.4 \mathrm{~Hz}, 1 \mathrm{H}, \mathrm{NH}\right), 6.45$ (t, $J=2.2 \mathrm{~Hz}, 1 \mathrm{H}$, Ar-H), 6.48 (d, $J=2.3 \mathrm{~Hz}, 1 \mathrm{H}, \mathrm{Ar}-\mathrm{H}), 6.61$ (d, $J=8.1 \mathrm{~Hz}, 1 \mathrm{H}, \operatorname{Ar}-\mathrm{H}), 7.37$ (dd, $J=6.5,2.1 \mathrm{~Hz}$, 2H, Ar-H), $7.50(\mathrm{~d}, J=8.4 \mathrm{~Hz}, 2 \mathrm{H}, \mathrm{Ar}-\mathrm{H}) .{ }^{13} \mathrm{C} \mathrm{NMR}: \delta 16.5(\mathrm{~d}, J=8.2 \mathrm{~Hz}), 55.9(\mathrm{~d}, J=151.3$ $\mathrm{Hz}), 65.0(\mathrm{~d}, J=6.5 \mathrm{~Hz}), 113.1,115.5,118.6,122.9,125.8,130.9,132.6,136.4,144.9 .{ }^{31} \mathrm{P}$ NMR : $\delta$ 22.32. Anal. Calcd for $\mathrm{C}_{17} \mathrm{H}_{20} \mathrm{BrClNO}_{4} \mathrm{P}: \mathrm{C}, 45.51 ; \mathrm{H}, 4.49 ; \mathrm{N}, 3.12$. Found : $\mathrm{C}, 45.68$; $\mathrm{H}, 4.43 ; \mathrm{N}, 3.09$.

[(5-Chloro-2-hydroxyphenylamino)-p-tolylmethyl]diethylphosphonate (4d). Yield (\%) : 69. mp : 148-150 ${ }^{\circ} \mathrm{C}$. IR : $742\left(\mathrm{P}-\mathrm{C}_{\text {aliphatic }}\right), 1198(\mathrm{P}=\mathrm{O}), 3399(\mathrm{~N}-\mathrm{H}) \mathrm{cm}^{-1} .{ }^{1} \mathrm{H}$ NMR : $\delta 1.28(\mathrm{t}, J=7.0$ $\left.\mathrm{Hz}, 3 \mathrm{H}, \mathrm{P}-\mathrm{OCH}_{2} \underline{\mathrm{C}}_{3}\right), 1.15\left(\mathrm{t}, J=7.0 \mathrm{~Hz}, 3 \mathrm{H}, \mathrm{P}-\mathrm{OCH}_{2} \underline{\mathrm{C}}_{3}\right), 2.30\left(\mathrm{~s}, 3 \mathrm{H}, \mathrm{CH}_{3}\right), 3.79-4.00$ $\left(\mathrm{m}, 4 \mathrm{H}, \mathrm{P}-\mathrm{OCH}_{2} \mathrm{CH}_{3}\right), 4.09-4.14(\mathrm{~m}, 1 \mathrm{H}, \mathrm{P}-\mathrm{CH}), 4.92(\mathrm{~s}, 1 \mathrm{H}, \mathrm{NH}), 6.43$ (d, $J=2.2 \mathrm{~Hz}, 2 \mathrm{H}, \mathrm{Ar}-$ H), $6.50(\mathrm{~d}, J=8.9 \mathrm{~Hz}, 1 \mathrm{H}, \mathrm{Ar}-\mathrm{H}), 7.16(\mathrm{~d}, J=7.8 \mathrm{~Hz}, 2 \mathrm{H}, \mathrm{Ar}-\mathrm{H}), 7.32$ (dd, $J=6.0,2.0 \mathrm{~Hz}, 2 \mathrm{H}$, Ar-H). ${ }^{13} \mathrm{C}$ NMR : $\delta 16.5(\mathrm{~d}, J=8.5 \mathrm{~Hz}), 21.1,56.2(\mathrm{~d}, J=152.1 \mathrm{~Hz}), 64.7(\mathrm{~d}, J=7.6 \mathrm{~Hz})$, 113.1, 115.4, 118.3, 125.8, 129.0, 130.2, 133.7, 139.1, 144.9. ${ }^{31} \mathrm{P}$ NMR : $\delta$ 23.32. Anal. Calcd for $\mathrm{C}_{18} \mathrm{H}_{23} \mathrm{ClNO}_{4} \mathrm{P}$ C, 56.33; H, 6.04; N, 3.65. Found : C, 56.44; H, 6.12; N, 3.58.

[(5-Chloro-2-hydroxyphenylamino)-(4-allyloxyphenyl)methyl]diethylphosphonate (4e). Yield (\%) : 67. mp : 120-122 ${ }^{\circ} \mathrm{C}$. IR : $747\left(\mathrm{P}-\mathrm{C}_{\text {aliphatic }}\right), 1161(\mathrm{P}=\mathrm{O}), 3388(\mathrm{~N}-\mathrm{H}) \mathrm{cm}^{-1} .{ }^{1} \mathrm{H} \mathrm{NMR}: \delta 1.28$ $\left(\mathrm{t}, J=7.0 \mathrm{~Hz}, 3 \mathrm{H}, \mathrm{P}-\mathrm{OCH}_{2} \mathrm{CH}_{3}\right), 1.56\left(\mathrm{t}, J=7.0 \mathrm{~Hz}, 3 \mathrm{H}, \mathrm{P}-\mathrm{OCH}_{2} \underline{\mathrm{C}}_{3}\right), 3.79-3.86(\mathrm{~m}, 4 \mathrm{H}$, P-OC $\left.\underline{H}_{2} \mathrm{CH}_{3}\right), 4.09-4.14(\mathrm{~m}, 1 \mathrm{H}, \mathrm{P}-\mathrm{CH}), 4.90(\mathrm{~s}, 1 \mathrm{H}, \mathrm{NH}),[4.51(\mathrm{~d}, J=5.1 \mathrm{~Hz}, 2 \mathrm{H}), 5.22(\mathrm{~d}$, $J=10.6 \mathrm{~Hz}, 1 \mathrm{H}), 5.37(\mathrm{~d}, J=17.2 \mathrm{~Hz}, 1 \mathrm{H}), 5.99-6.07(\mathrm{~m}, 1 \mathrm{H})]$ allyloxy-H, $6.44(\mathrm{~d}, J=2.3 \mathrm{~Hz}$, $1 \mathrm{H}, \mathrm{Ar}-\mathrm{H}), 6.45$ (d, $J=9.1 \mathrm{~Hz}, 1 \mathrm{H}, \mathrm{Ar}-\mathrm{H}), 6.59$ (d, $J=8.0 \mathrm{~Hz}, 1 \mathrm{H}, \mathrm{Ar}-\mathrm{H}), 6.91$ (d, $J=8.4 \mathrm{~Hz}$, 2H, Ar-H), 7.35 (dd, $J=6.5,2.0 \mathrm{~Hz}, 2 \mathrm{H}, \mathrm{Ar}-\mathrm{H}) .{ }^{13} \mathrm{C} \mathrm{NMR}: \delta 16.6(\mathrm{~d}, J=9.0 \mathrm{~Hz}), 55.8(\mathrm{~d}, J=$ 
$153.0 \mathrm{~Hz}), 64.7(\mathrm{~d}, J=6.9 \mathrm{~Hz}), 69.9,113.1,115.4,115.9,117.5,118.3,125.8,128.8,130.2$, 134.8, 144.9, 160.0. ${ }^{31} \mathrm{P}$ NMR : $\delta$ 23.45. Anal. Calcd for $\mathrm{C}_{20} \mathrm{H}_{25} \mathrm{ClNO}_{5} \mathrm{P}: \mathrm{C}, 56.41 ; \mathrm{H}, 5.92 ; \mathrm{N}$, 3.29. Found: C, $56.38 ; \mathrm{H}, 5.88 ; \mathrm{N}, 3.24$.

[(5-Chloro-2-hydroxyphenylamino)-(4-methoxyphenyl)methyl]diethylphosphonate (4f). Yield (\%) : 76. mp : 177-179 ${ }^{\circ} \mathrm{C}$. IR : 754 (P-C aliphatic), $1247(\mathrm{P}=\mathrm{O}), 3382(\mathrm{~N}-\mathrm{H}) \mathrm{cm}^{-1} .{ }^{1} \mathrm{H}$ NMR : $\delta 1.27$ (t, $\left.J=7.0 \mathrm{~Hz}, 3 \mathrm{H}, \mathrm{P}-\mathrm{OCH}_{2} \mathrm{CH}_{3}\right), 1.14\left(\mathrm{t}, J=7.0 \mathrm{~Hz}, 3 \mathrm{H}, \mathrm{P}-\mathrm{OCH}_{2} \mathrm{CH}_{3}\right), 3.81-4.00(\mathrm{~m}, 4 \mathrm{H}$, P-OC $\left.\underline{H}_{2} \mathrm{CH}_{3}\right), 3.76\left(\mathrm{~s}, 3 \mathrm{H}, \mathrm{OCH}_{3}\right), 4.09-4.15(\mathrm{~m}, 1 \mathrm{H}, \mathrm{P}-\mathrm{CH}), 4.90(\mathrm{~s}, 1 \mathrm{H}, \mathrm{NH}), 6.43(\mathrm{~d}, J=2.2$ $\mathrm{Hz}, 1 \mathrm{H}, \mathrm{Ar}-\mathrm{H}), 6.44$ (d, $J=8.0 \mathrm{~Hz}, 1 \mathrm{H}, \mathrm{Ar}-\mathrm{H}), 6.59$ (d, $J=7.9 \mathrm{~Hz}, 1 \mathrm{H}, \mathrm{Ar}-\mathrm{H}), 6.89$ (d, $J=8.5$ $\mathrm{Hz}, 2 \mathrm{H}, \mathrm{Ar}-\mathrm{H}), 7.35$ (dd, $J=6.5,2.1 \mathrm{~Hz}, 2 \mathrm{H}, \mathrm{Ar}-\mathrm{H}) .{ }^{13} \mathrm{C}$ NMR $: \delta 16.7,55.8(\mathrm{~d}, J=153.4 \mathrm{~Hz})$, 56.1, 65.0, 113.2, 115.2, 115.7, 118.6, 128.5, 130.2. ${ }^{31} \mathrm{P}$ NMR : $\delta$ 26.77. Anal. Calcd for $\mathrm{C}_{18} \mathrm{H}_{23} \mathrm{ClNO}_{5} \mathrm{P}: \mathrm{C}, 54.07 ; \mathrm{H}, 5.80 ; \mathrm{N}, 3.50$. Found : C, 54.17; H, 5.75; N, 3.48 .

[(5-Chloro-2-hydroxyphenylamino)-(3,4-dimethoxyphenyl)methyl]diethylphosphonate (4g). Yield (\%) : 82. mp : 109-111 ${ }^{\circ} \mathrm{C}$. IR : $752\left(\mathrm{P}-\mathrm{C}_{\text {aliphatic }}\right), 1264(\mathrm{P}=\mathrm{O}), 3372(\mathrm{~N}-\mathrm{H}) \mathrm{cm}^{-1}$. ${ }^{1} \mathrm{H}$ NMR : $\delta 1.29\left(\mathrm{t}, J=7.0 \mathrm{~Hz}, 3 \mathrm{H}, \mathrm{P}-\mathrm{OCH}_{2} \mathrm{C}_{3}\right), 1.17$ (t, $\left.J=7.0 \mathrm{~Hz}, 3 \mathrm{H}, \mathrm{P}-\mathrm{OCH}_{2} \mathrm{CH}_{3}\right), 3.80$ (s, $3 \mathrm{H}$, $\left.\mathrm{OCH}_{3}\right), 3.82\left(\mathrm{~s}, 3 \mathrm{H}, \mathrm{OCH}_{3}\right), 3.84-4.03\left(\mathrm{~m}, 4 \mathrm{H}, \mathrm{P}-\mathrm{OCH}_{2} \mathrm{CH}_{3}\right), 4.11-4.16(\mathrm{~m}, 1 \mathrm{H}, \mathrm{P}-\mathrm{CH}), 4.92(\mathrm{~s}$, 1H, NH), 6.45 (dd, $J=6.5,2.3 \mathrm{~Hz}, 1 \mathrm{H}, \mathrm{Ar}-\mathrm{H}), 6.48$ (d, $J=9.0 \mathrm{~Hz}, 1 \mathrm{H}, \mathrm{Ar}-\mathrm{H}), 6.60$ (d, $J=8.2$ $\mathrm{Hz}, 1 \mathrm{H}, \mathrm{Ar}-\mathrm{H}), 6.91-7.06$ (m, 3H, Ar-H). ${ }^{31} \mathrm{P} \mathrm{NMR}: \delta$ 26.67. Anal. Calcd for $\mathrm{C}_{19} \mathrm{H}_{25} \mathrm{ClNO}_{6} \mathrm{P}: \mathrm{C}$, 53.09; H, 5.86; N, 3.26. Found : C, 53.12; H, 5.78; N, 3.17.

[(5-Chloro-2-hydroxyphenylamino)-phneylmethyl]diethylphosphonate (4h). Yield (\%): 81. mp : 125-127 ${ }^{\circ} \mathrm{C}$. IR : $758\left(\right.$ P-C $\left._{\text {aliphatic }}\right), 1216(\mathrm{P}=\mathrm{O}), 3404(\mathrm{~N}-\mathrm{H}) \mathrm{cm}^{-1} .{ }^{1} \mathrm{H}$ NMR : $\delta 1.28$ (t, $J=7.0$ $\left.\mathrm{Hz}, 3 \mathrm{H}, \mathrm{P}-\mathrm{OCH}_{2} \underline{\mathrm{C}}_{3}\right), 1.15$ (t, $\left.J=7.0 \mathrm{~Hz}, 3 \mathrm{H}, \mathrm{P}-\mathrm{OCH}_{2} \underline{\mathrm{C}}_{3}\right), 3.79-4.01\left(\mathrm{~m}, 4 \mathrm{H}, \mathrm{P}-\mathrm{OC}_{2} \mathrm{CH}_{3}\right)$, 4.11-4.15 (m, 1H, P-CH), 4.95 (d, $J=24.2 \mathrm{~Hz}, 1 \mathrm{H}, \mathrm{NH}), 6.43$ (d, $J=2.3 \mathrm{~Hz}, 1 \mathrm{H}, \mathrm{Ar}-\mathrm{H}), 6.45$ $(\mathrm{d}, J=6.5 \mathrm{~Hz}, 1 \mathrm{H}, \mathrm{Ar}-\mathrm{H}), 6.60(\mathrm{~d}, J=8.9 \mathrm{~Hz}, 1 \mathrm{H}, \mathrm{Ar}-\mathrm{H}), 7.26-7.46(\mathrm{~m}, 5 \mathrm{H}, \mathrm{Ar}-\mathrm{H}) .{ }^{31} \mathrm{P}$ NMR $: \delta$ 26.44. Anal. Calcd for $\mathrm{C}_{17} \mathrm{H}_{21} \mathrm{ClNO}_{4} \mathrm{P}: \mathrm{C}, 55.22 ; \mathrm{H}, 5.72 ; \mathrm{N}, 3.79$. Found : $\mathrm{C}, 55.13 ; \mathrm{H}, 5.63 ; \mathrm{N}$, 3.68 .

[(5-Chloro-2-hydroxyphenylamino)-thiophen-2-yl-methyl]diethylphosphonate (4i). Yield (\%): 66. $\mathrm{mp}: 105-107^{\circ} \mathrm{C}$. IR : 751 (P-C $\left.\mathrm{C}_{\text {aliphatic }}\right), 1214(\mathrm{P}=\mathrm{O}), 3348(\mathrm{~N}-\mathrm{H}) \mathrm{cm}^{-1} .{ }^{1} \mathrm{H}$ NMR : $\delta 1.28(\mathrm{t}$, $\left.J=7.0 \mathrm{~Hz}, 3 \mathrm{H}, \mathrm{P}-\mathrm{OCH}_{2} \mathrm{C}_{3}\right), 1.20\left(\mathrm{t}, J=7.0 \mathrm{~Hz}, 3 \mathrm{H}, \mathrm{P}-\mathrm{OCH}_{2} \mathrm{C}_{3}\right), 3.91-4.09$ (m, 4H, P$\left.\mathrm{OC}_{2} \mathrm{CH}_{3}\right), 4.11-4.19(\mathrm{~m}, 1 \mathrm{H}, \mathrm{P}-\mathrm{CH}), 5.28(\mathrm{~d}, J=23.6 \mathrm{~Hz}, 1 \mathrm{H}, \mathrm{NH}), 6.49$ (dd, $J=6.2,2.3 \mathrm{~Hz}$, $1 \mathrm{H}, \mathrm{Ar}-\mathrm{H}), 6.1(\mathrm{~d}, J=8.3 \mathrm{~Hz}, 1 \mathrm{H}, \mathrm{Ar}-\mathrm{H}), 6.63(\mathrm{~d}, J=2.2 \mathrm{~Hz}, 1 \mathrm{H}, \mathrm{Ar}-\mathrm{H}), 6.98-7.34$ (m, 3H, ArH). ${ }^{31} \mathrm{P}$ NMR: $\delta$ 22.30. Anal. Calcd for $\mathrm{C}_{15} \mathrm{H}_{19} \mathrm{ClNO}_{4} \mathrm{PS}: \mathrm{C}, 47.94 ; \mathrm{H}, 5.10 ; \mathrm{N}, 3.73$. Found : $\mathrm{C}$, $48.06 ; \mathrm{H}, 5.17 ; \mathrm{N}, 3.80$.

[(5-Chloro-2-hydroxyphenylamino)-(3-hydroxy-4-methoxyphenyl)methyl]diethylphosphonate (4j). Yield (\%): 70. mp : 143-145 ${ }^{\circ} \mathrm{C}$. IR : 745 (P-C aliphatic), $1196(\mathrm{P}=\mathrm{O}), 3359(\mathrm{~N}-\mathrm{H}) \mathrm{cm}^{-1} .{ }^{1} \mathrm{H}$ NMR : $\delta 1.28\left(\mathrm{t}, J=7.0 \mathrm{~Hz}, 3 \mathrm{H}, \mathrm{P}-\mathrm{OCH}_{2} \underline{\mathrm{C}}_{3}\right), 1.17\left(\mathrm{t}, J=7.0 \mathrm{~Hz}, 3 \mathrm{H}, \mathrm{P}-\mathrm{OCH}_{2} \underline{\mathrm{C}}_{3}\right), 3.83(\mathrm{~s}, 3 \mathrm{H}$, $\left.\mathrm{OCH}_{3}\right), 3.95-4.01\left(\mathrm{~m}, 4 \mathrm{H}, \mathrm{P}-\mathrm{OC} \underline{H}_{2} \mathrm{CH}_{3}\right), 4.11-4.18(\mathrm{~m}, 1 \mathrm{H}, \mathrm{P}-\mathrm{CH}), 4.84(\mathrm{~s}, 1 \mathrm{H}, \mathrm{NH}), 6.45$ (dd, $J=6.1,2.0 \mathrm{~Hz}, 1 \mathrm{H}, \operatorname{Ar}-\mathrm{H}), 6.50(\mathrm{~s}, 1 \mathrm{H}, \operatorname{Ar}-\mathrm{H}), 6.60(\mathrm{~d}, J=8.2 \mathrm{~Hz}, 1 \mathrm{H}, \operatorname{Ar}-\mathrm{H}), 6.77(\mathrm{~d}$, $J=7.9 \mathrm{~Hz}, 1 \mathrm{H}, \mathrm{Ar}-\mathrm{H}), 6.88(\mathrm{~d}, J=6.0 \mathrm{~Hz}, 1 \mathrm{H}, \mathrm{Ar}-\mathrm{H}), 6.89(\mathrm{~d}, J=2.0 \mathrm{~Hz}, 1 \mathrm{H}, \mathrm{Ar}-\mathrm{H}), 7.03(\mathrm{~s}$, $1 \mathrm{H}$, Ar-H). ${ }^{31} \mathrm{P}$ NMR: $\delta$ 26.94. Anal. Calcd for $\mathrm{C}_{18} \mathrm{H}_{23} \mathrm{ClNO}_{6} \mathrm{P}: \mathrm{C}, 51.99 ; \mathrm{H}, 5.58 ; \mathrm{N}, 3.37$. Found : C, 52.09; H, 5.56; N, 3.43 . 
[(5-Chloro-2-hydroxyphenylamino)-(3-hydroxyphenyl)methyl]diethylphosphonate

(4k).

Yield (\%): 74. mp : $128-130^{\circ} \mathrm{C}$. IR : $756\left(\mathrm{P}-\mathrm{C}_{\text {aliphatic }}\right), 1201(\mathrm{P}=\mathrm{O}), 3314(\mathrm{~N}-\mathrm{H}) \mathrm{cm}^{-1} .{ }^{1} \mathrm{H}$ NMR : $\delta 1.28\left(\mathrm{t}, J=7.0 \mathrm{~Hz}, 3 \mathrm{H}, \mathrm{P}-\mathrm{OCH}_{2} \underline{\mathrm{C}}_{3}\right), 1.66$ (t, $\left.J=7.0 \mathrm{~Hz}, 3 \mathrm{H}, \mathrm{P}-\mathrm{OCH}_{2} \mathrm{C}_{3}\right), 3.80-4.01$ (m, 4H, P-OC $\left.\underline{H}_{2} \mathrm{CH}_{3}\right), 4.11-4.16$ (m, 1H, P-CH), 4.84 (s, 1H, NH), 6.45 (d, J=2.0 Hz, 2H, Ar-H), 6.59 $(\mathrm{d}, J=8.7 \mathrm{~Hz}, 1 \mathrm{H}, \mathrm{Ar}-\mathrm{H}), 6.70-7.17(\mathrm{~m}, 4 \mathrm{H}, \mathrm{Ar}-\mathrm{H}) .{ }^{31} \mathrm{P}$ NMR: $\delta$ 22.60. Anal. Calcd for $\mathrm{C}_{17} \mathrm{H}_{21} \mathrm{ClNO}_{5} \mathrm{P}: \mathrm{C}, 52.93 ; \mathrm{H}, 5.49 ; \mathrm{N}, 3.63$. Found : C, 53.06; H, 5.55; N, 3.70.

[(5-Chloro-2-hydroxyphenylamino)-(2-hydroxyphenyl)methyl]diethylphosphonate (4I). Yield (\%): 65. mp : $134-135^{\circ} \mathrm{C}$. IR : $754\left(\mathrm{P}-\mathrm{C}_{\text {aliphatic }}\right), 1191(\mathrm{P}=\mathrm{O}), 3274(\mathrm{~N}-\mathrm{H}) \mathrm{cm}^{-1}$. ${ }^{1} \mathrm{H}$ NMR : $\delta 1.30\left(\mathrm{t}, J=7.0 \mathrm{~Hz}, 3 \mathrm{H}, \mathrm{P}-\mathrm{OCH}_{2} \underline{\mathrm{C}}_{3}\right), 1.11$ (t, $\left.J=7.0 \mathrm{~Hz}, 3 \mathrm{H}, \mathrm{P}-\mathrm{OCH}_{2} \mathrm{C}_{3}\right), 3.79-4.00(\mathrm{~m}, 4 \mathrm{H}$, P-OC $\left.\underline{H}_{2} \mathrm{CH}_{3}\right), 4.13-4.18(\mathrm{~m}, 1 \mathrm{H}, \mathrm{P}-\mathrm{CH}), 5.32(\mathrm{~d}, J=24.2 \mathrm{~Hz}, 1 \mathrm{H}, \mathrm{NH}), 6.42(\mathrm{dd}, J=6.0,2.3 \mathrm{~Hz}$, $1 \mathrm{H}, \mathrm{Ar}-\mathrm{H}), 6.51$ (d, $J=2.3 \mathrm{~Hz}, 1 \mathrm{H}, \mathrm{Ar}-\mathrm{H}), 6.57$ (d, $J=8.2 \mathrm{~Hz}, 1 \mathrm{H}, \mathrm{Ar}-\mathrm{H}), 6.77-7.31$ (m, 4H, ArH). ${ }^{31} \mathrm{P}$ NMR: $\delta$ 27.44. Anal. Calcd for $\mathrm{C}_{17} \mathrm{H}_{21} \mathrm{ClNO}_{5} \mathrm{P}: \mathrm{C}, 52.93 ; \mathrm{H}, 5.49 ; \mathrm{N}, 3.63$. Found : $\mathrm{C}$, 53.04; H, 5.54; N, 3.71.

\section{Disc diffusion bioassay}

Suspension of approximately $1.5 \times 10^{8}$ bacterial cells per $\mathrm{mL}$ were used. In sterile normal saline was prepared as described by Forbes $e t a l^{15}$ and $1.5 \mathrm{~mL}$ of it was uniformly spreaded on Nutrient Agar (Hi-media) in 12 x $1.2 \mathrm{~cm}$ glass Petri dishes, left aside for $15 \mathrm{~min}$ and excess of suspension was then drained and discarded. For the agar disc diffusion method, the test compound was introduced onto the disc and then allowed to dry to completely saturate the disc with the test compound. Then the disc was introduced onto the upper layer of the medium with the bacteria. The petridishes were incubated overnight at $37^{\circ} \mathrm{C}$ for $24 \mathrm{hrs}$. Bioactivity was determined by measuring Diameter of Inhibition Zones (DIZ) in mm. The compounds 4a-l were taken as 20 and $40 \mu \mathrm{g} / \mathrm{mL}$ concentrations and were evaluated by disc method. Each test was done in triplicate and the mean of the diameter of the inhibition zones was calculated. Controls included the use of solvent without test compounds although no antibacterial activity was noted for the solvent employed in the test. ${ }^{16}$

\section{Determination of minimum inhibitory concentration (MIC)}

The lowest concentration (highest dilution) of the compounds 4a-l that produced no visible bacterial growth (no turbidity) when compared with the control tubes ${ }^{16}$ MIC was determined for the compounds 4a-l. Compounds $4 \mathbf{a}-\mathbf{l}$ in concentrations of $0.1-4.1 \mathrm{mg} / \mathrm{mL}$ in steps of $100 \mu \mathrm{g} /$ $\mathrm{mL}$ were evaluated. Specifically $0.1 \mathrm{~mL}$ of standardized inoculum $\left(1-2 \times 10^{7} \mathrm{CFU} / \mathrm{mL}\right)$ was added to each tube. The tubes were incubated aerobically at $37^{\circ} \mathrm{C}$ for $18-24 \mathrm{hr}$. Two control tubes were maintained for each test batch. These included antibiotic control (tube containing compounds 4a-l and the growth medium without inoculum) and organism control (the tube containing the growth medium, physiological saline and the inoculum). 


\section{Conclusions}

We synthesized new $\alpha$-aminophosphonates $4 a-1$ in high yields by Kabachnik-Fields reaction without using any catalyst. All of them showed low antibacterial activity against selected bacteria, while compounds 4a exhibited significant antibacterial activity even at lower concentration against both Gram negative and Gram positive bacteria. X-ray crystallographic studies for $\mathbf{4 g}$ proved distorted tetrahedral configuration for $\mathrm{P}$ in these compounds.

\section{Acknowledgements}

The authors express thanks to Prof. C. Devendranath Reddy and Dr. C. Naga Raju, Dept. of Chemistry, S. V. University, Tirupati, for helpful discussions and SIF, IISc, Bangalore, for providing the NMR spectra.

\section{References}

1. Drake, G. L.; Culamaria, T. A. The Role of Phosphonates in Living Systems, Hilderbrand, R. L. Ed., CRC Press, 1983, Chapter 7.

2. (a) Fields, S. C. Tetrahedron 1999, 55, 12237. (b) Redmore, D. J. Org. Chem. 1978, 43, 992.

3. Kafarski, P.; Lejczak, B. Phosphorus Sulfur Silicon, Relat. Elem. 1991, 63, 1993.

4. Allen, M. C.; Fuhrer, W.; Tuck, B.; Wade R.; Wood, J. M. J. Med. Chem. 1989, 32, 1652.

5. Hirschmann, R.; Smith, A. B. III.; Taylor, C. M.; Benkovic, P. A.; Taylor, S. D.; Yager, K. M.; Spengler, P. A.; Venkovic, S. J. Science 1994, 265, 234,.

6. (a) Baylis, E. K.; Campbell, C. D.; Dingwall, J. G. J. Chem. Soc., Perkin Trans. 1984, 1, 2845. (b) Atherton, F. R.; Hassall, C. H.; Lambert, R. W. J. Med. Chem. 1986, $29,29$.

7. Miliszkiewicz, D.; Wieczorek, P.; Lejczak, B.; Kowalik, E.; Kafarski, P. Pestic Sci. 1992, 34, 349.

8. (a) Kukhar, V. P.; Solodenko, V. A. Rus. Chem. Rev (Engl. Trans.). 1987, 56, 859. (b) Yokomatsu, T.; Yoshida, Y.; Shibuya, S. J. Org. Chem. 1994, 59, 7930.

9. Silverstein, R. M.; Bassler, G. C.; Morill, T. C. Spectrometric Identification of Organic Compounds, 6th Ed., John Wiley \& Sons: New York, 1991.

10. (a) Yadav, J. S.; Reddy, B. V. S.; Sarita Raj, K.; Bhaskar Reddy, K.; Prasad, A. R.; Synthesis 2001, 2277. (b) Firouzabadi, H.; Iranpoor, N.; Sobhani, S. Synthesis 2004, 2692.

11. Quin, L. D.; Verkade, J. G. Phosphorus-31NMR Spectral Properties in Compound Characterization and Structural Analysis, VCH P: New York, 1994.

12. Sheldrick, G. M. SHELX-97, University of Gottingen, 1997.

13. Sasada, Y. Molecular and Crystal Structures. In Chemistry Handbook, 3rd Ed., Tokyo, The Chemical Society of Japan, Maruzen, 1984. 
14. Wang, Z. X.; Jian, F. F.; Duan, C. Y.; Bai, Z. P.; You, X. Z. Acta Cryst. C54, 1998, 1927.

15. Forbes B. A.; Sahm, O. F.; Weissfeld, A. S.; Trevomp, E. A. Methods for Testing Antimicrobial Effectiveness. In Bailey and Scott's Diagnostic Microbiology MOS by Co: St Louis, Missouri, 1990; p 171.

16. Shahidi Bonjar, G. H. Asian J. Plant Sci. 2004, 3, 56. 